\title{
Differing Effect of the Sodium-Glucose Cotransporter 2 Inhibitor Ipragliflozin on the Decrease of Fat Mass vs. Lean Mass in Patients With or Without Metformin Therapy
}

\author{
Yoshio Nagai ${ }^{a}$, b, Hisashi Fukuda ${ }^{a}$, Shin Kawanabea, Tomoko Nakagawa ${ }^{a}$, \\ Akio Ohta ${ }^{\text {a, }}$ Yasushi Tanaka ${ }^{\text {a }}$
}

\begin{abstract}
Background: We previously reported changes of body composition determined by dual-energy X-ray absorptiometry after treatment with ipragliflozin, a sodium-glucose cotransporter 2 (SGLT2) inhibitor. In that study, mean body weight was decreased by $3.5 \mathrm{~kg}(4.3 \%$ of the baseline value) after ipragliflozin treatment at $50 \mathrm{mg} /$ day, with fat mass and lean mass showing similar reductions of 1.7 and $1.8 \mathrm{~kg}$, respectively. A long-term decrease of lean mass in patients treated with SGLT2 inhibitors may be associated with loss of skeletal muscle, which could potentially have an impact on quality of life.
\end{abstract}

Methods: In this post hoc analysis, we investigated whether changes of body composition were influenced by other medications for diabetes in 20 patients (11 men and nine women) who received ipragliflozin for 24 weeks.

Results: When we divided the patients into two subgroups with or without metformin treatment, fat mass showed a significant decrease in the ipragliflozin + metformin subgroup and a significantly greater decrease compared to the ipragliflozin subgroup $(2.0 \mathrm{~kg}$; $95 \%$ confidence interval (CI): $0.1-3.9 ; \mathrm{P}=0.038)$. Lean mass was significantly decreased in the ipragliflozin subgroup, but the decrease showed no significant difference from that in the ipragliflozin + metformin subgroup ( $1.9 \mathrm{~kg} ; 95 \% \mathrm{CI}:-4.1-0.3 ; \mathrm{P}=0.087)$. No significant differences of body composition changes were observed with other antidiabetic agents.

Conclusions: More desirable weight reduction due to preferential fat loss and less muscle loss may be achieved by combining an SGLT2 inhibitor with metformin.

Manuscript submitted February 14, 2019, accepted February 27, 2019

aDivision of Metabolism and Endocrinology, Department of Internal Medicine, St. Marianna University School of Medicine, Kawasaki 216-8511, Japan ${ }^{\mathrm{b}}$ Corresponding Author: Yoshio Nagai, Division of Metabolism and Endocrinology, Department of Internal Medicine, St. Marianna University School of Medicine, 2-16-1, Sugao, Miyamae-ku, Kawasaki, Kanagawa 216-8511, Japan. Email: ynagai@marianna-u.ac.jp

doi: https://doi.org/10.14740/jocmr3785
Keywords: Body composition; Ipragliflozin; Metformin; Type 2 diabetes mellitus

\section{Introduction}

Sodium-glucose cotransporter 2 (SGLT2) inhibitors are oral antidiabetic agents that decrease the blood glucose level by enhancing urinary glucose excretion. Reduction of body weight as well as blood glucose is observed because of energy loss due to urinary excretion of $60-80 \mathrm{~g}$ of glucose daily [1].

We previously investigated changes of body composition by using dual-energy X-ray absorptiometry (DXA) in obese Japanese patients with type 2 diabetes mellitus (T2DM) who received treatment with the SGLT2 inhibitor ipragliflozin for 24 weeks [2]. In that study, mean body weight was decreased by $3.5 \mathrm{~kg}(4.3 \%$ of the baseline value $)$ after ipragliflozin treatment at $50 \mathrm{mg} /$ day, with fat mass (FM) and lean mass (LM) showing similar reductions of 1.7 and $1.8 \mathrm{~kg}$, respectively. LM includes body water, but SGLT2 inhibitors only cause transient reduction of plasma volume that is largely reversed after 12 weeks [3]. Thus, a long-term decrease of LM in patients treated with SGLT2 inhibitors may be associated with loss of skeletal muscle. Weight reduction obtained by treatment with SGLT2 inhibitors has been reported to be beneficial in patients with metabolic disorders, such as hypertension, nonalcoholic fatty liver disease, and hyperuricemia [2]. However, a decrease of muscle mass may be related to frailty, which is a major cause of physical dysfunction, morbidity, and mortality in the elderly. In Japan, the number of elderly patients with T2DM continues to increase, and $33.7 \%$ of patients with T2DM were over 70 years old according to the 2016 Japan National Health and Nutrition Survey [4]. The objectives of treating diabetes are to prevent the onset and progression of diabetic complications through tight glycemic and metabolic control, as well as to maintain the quality of life (QOL) and longevity. However, reduction of muscle mass associated with SGLT2 inhibitor therapy could potentially have an impact on QOL.

Accordingly, we performed a post hoc analysis of data collected prospectively from patients administered the SGLT2 
inhibitor ipragliflozin to investigate whether changes of body composition were influenced by their other treatment for diabetes.

\section{Materials and Methods}

This analysis assessed data from 20 patients enrolled in the prospective, 24-week, single-center, open-label, single-arm study conducted at St. Marianna University Hospital (Kawasaki, Japan) between July 2014 and June 2015. The primary objectives were to clarify the effects of ipragliflozin $(50 \mathrm{mg} /$ day) on body composition parameters (assessed by DXA), abdominal visceral and subcutaneous fat volume (assessed by whole abdominal CT scanning), and the intrahepatic lipid content (determined by proton magnetic resonance spectroscopy). The secondary objective was to investigate the effect of ipragliflozin on beta-cell stress, which was assessed from the proinsulin/C-peptide ratio. The results related to these objectives have been published previously $[2,5]$. The present post hoc subgroup analysis investigated the influence of antidiabetic drugs other than ipragliflozin.

The protocol for this research project has been approved by a suitably constituted Ethics Committee of St. Marianna University School of Medicine and it conforms to the provisions of the Declaration of Helsinki (approval No. 2678, approval date: June 24, 2014). All informed consent was obtained from the subject. This study was registered with the University Hospital Medical Network Clinical Trials Registry (clinical trial registration number: UMIN000021623).

\section{Statistical analysis}

Continuous variables were expressed as the mean \pm standard deviation (SD) and variables with a normal distribution were compared by using the $t$-test. Skewed variables were expressed as the median (interquartile range (IQR)) and were compared with Wilcoxon's signed rank test. All analyses were performed with SPSS 21.0 software and $\mathrm{P}<0.05$ was accepted as indicating a significant difference.

\section{Results}

The 20 patients (11 men and nine women) who completed the study were aged $51.6 \pm 8.0$ years, and they had a baseline body weight of $82.2 \pm 11.3 \mathrm{~kg}$, body mass index (BMI) of $29.7 \pm$ $3.2 \mathrm{~kg} / \mathrm{m}^{2}$, and hemoglobin A1c (HbA1c) of $8.2 \pm 1.3 \%$. Before starting ipragliflozin, the patients were being treated with a biguanide (metformin) $(\mathrm{n}=11)$, insulin $(\mathrm{n}=9)$, sulfonylurea $(\mathrm{n}$ $=8)$, DPP-4 inhibitor $(\mathrm{n}=7)$, GLP-1 receptor agonist $(\mathrm{n}=5)$, $\alpha$-glucosidase inhibitor $(n=4)$, or thiazolidinedione (pioglitazone) $(n=3)$. None of the patients developed hypoglycemia, which meant that their other antidiabetic therapy (including insulin dosages) was not changed during the study period. We divided the patients into two subgroups with or without metformin, which was the other antidiabetic drug used most fre- quently in the study population.

Baseline clinical characteristics of the two subgroups were well balanced with regard to determinants of metformin use, including the gender, age, duration of diabetes, BMI, HbAlc, and estimated glomerular filtration rate (Table 1). The median dose of metformin was 1,250 mg/day (1,000 - 1,500 mg/day), which was about half of the maximum dose approved in Japan $(2,250 \mathrm{mg})$. Mean body weight showed a significant decrease from baseline at 24 weeks in both subgroups (both $\mathrm{P}<0.01$ ), but there was no significant difference of weight reduction between the two subgroups. However, FM decreased significantly by $2.7 \mathrm{~kg}$ in the ipragliflozin + metformin subgroup compared with a decrease of only $0.7 \mathrm{~kg}$ in the ipragliflozin subgroup, and the reduction of FM showed a significant difference of $2.0 \mathrm{~kg}$ (95\% confidence interval (CI): $0.1-3.9 ; \mathrm{P}=$ 0.038 ) between the subgroups. In contrast, LM decreased by $3.0 \mathrm{~kg}$ in the ipragliflozin subgroup compared with a decrease of only $1.1 \mathrm{~kg}$ in the ipragliflozin + metformin subgroup, although the difference of $1.9 \mathrm{~kg}(95 \% \mathrm{CI}:-4.1-0.3)$ between the subgroups was not significant $(\mathrm{P}=0.087)$ (Fig. 1).

After 24 weeks of ipragliflozin treatment, HbA1c showed a significant decrease of $1.2 \%$ in both subgroups, and the difference of $0.12 \%(95 \% \mathrm{CI}: 0.90-0.86)$ between the two subgroups was not significant $(\mathrm{P}=0.946)$. The abdominal visceral fat volume and subcutaneous fat volume (on CT scans), as well as the intrahepatic lipid content (on MRI), also showed similar changes in the two subgroups. Apart from metformin, there were no significant differences in the changes of body composition when the patients were divided into subgroups according to their use of other antidiabetic agents with ipragliflozin (data not shown).

\section{Discussion}

The findings of this study suggested that the effect of the SGLT2 inhibitor ipragliflozin on body composition parameters differed between patients with or without concomitant metformin therapy. Reduction of FM (measured by DXA) accounted for about $75 \%$ of total weight loss in the ipragliflozin + metformin subgroup, which was consistent with a previous report on the effect of combined therapy with metformin and dapagliflozin [6]. However, that study did not provide comparative data regarding the changes of body composition with dapagliflozin monotherapy because the control group received placebo on background metformin therapy. According to American Diabetes Association/European Association for the Study of Diabetes and International Diabetes Federation [7-9], metformin is recommended as a first-line treatment for T2DM because of its favorable effect on body weight, low risk of hypoglycemia, and low cost. In Japan, it is suggested that glucose-lowering agents should be selected by considering the condition of each patient along with the pharmacological and safety profile of each drug [10]. Under these circumstances, metformin has not been widely used in Japan $[11,12]$, probably due to concern about serious adverse effects such as lactic acidosis and the need to monitor renal function or withdraw treatment when using contrast agents. This situation, in which metformin is gen- 
Table 1. Baseline Characteristics of the Subgroups Receiving Ipragliflozin Alone or With Metformin

\begin{tabular}{|c|c|c|c|}
\hline & Ipragliflozin alone $(n=9)$ & Ipragliflozin with metformin $(\mathrm{n}=11)$ & P value \\
\hline Women & 3 & 6 & 0.369 \\
\hline Age (years) & $53 \pm 10$ & $51 \pm 7$ & 0.626 \\
\hline Body mass index $\left(\mathrm{kg} / \mathrm{m}^{2}\right)$ & $29 \pm 2.3$ & $30.2 \pm 3.8$ & 0.416 \\
\hline Duration of diabetes (years) & $3(1-6)$ & $8(6.5-12)$ & 0.079 \\
\hline Fasting plasma glucose (mg/dL) & $161 \pm 17$ & $181 \pm 50$ & 0.288 \\
\hline Systolic blood pressure $(\mathrm{mm} \mathrm{Hg})$ & $139 \pm 11$ & $130 \pm 17$ & 0.207 \\
\hline Diastolic blood pressure (mm Hg) & $82 \pm 12$ & $80 \pm 15$ & 0.767 \\
\hline eGFR $\left(\mathrm{mL} / \mathrm{min} / 1.73 \mathrm{~m}^{2}\right)$ & $88 \pm 11$ & $92 \pm 11$ & 0.437 \\
\hline LDL-C (mg/dL) & $127 \pm 22$ & $105 \pm 27$ & 0.057 \\
\hline HDL-C (mg/dL) & $50 \pm 11$ & $46 \pm 7$ & 0.373 \\
\hline Sulfonylurea & 3 & 4 & \\
\hline Thiazolidinedione & 1 & 2 & \\
\hline$\alpha$-Glucosidase inhibitor & 0 & 4 & \\
\hline DPP-4 inhibitor & 3 & 4 & \\
\hline Insulin & 4 & 4 & \\
\hline GLP-1 receptor agonist & 0 & 4 & \\
\hline
\end{tabular}

Data are expressed as the mean \pm standard deviation for continuous variables or as the median (IQR). Categorical variables are expressed as numbers. eGFR: estimated glomerular filtration rate; LDL-C: low-density lipoprotein cholesterol; HDL-C: high-density lipoprotein cholesterol; DPP-4: dipeptidyl peptidase-4; GLP-1: glucagon-like peptide-1; IQR: interquartile range.

erally not a first-line treatment for T2DM in Japan, prompted us to investigate whether the effect of ipragliflozin on body

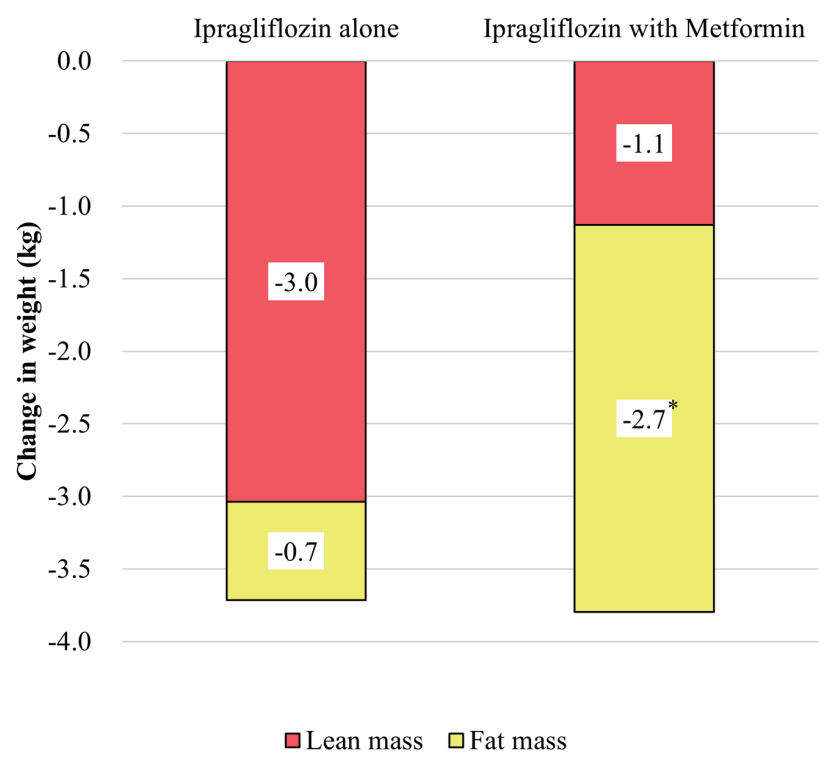

Figure 1. Changes of fat mass and lean mass assessed by dual energy X-ray absorptiometry during the 24-week study period. composition differed between patients with or without concomitant metformin therapy. Our study findings suggest that ipragliflozin creates an energy deficit, which is preferentially compensated from fat in patients also taking metformin, or preferentially compensated from muscle in patients not taking metformin. The mechanisms underlying our present finding are uncertain. A longitudinal cohort study of nearly 4,000 men with measurement of body composition parameters by DXA provided data suggesting that insulin sensitizers $(80 \%$ of the subjects received metformin) may ameliorate muscle loss [13]. One of the targets of metformin is AMP-activated protein kinase (AMPK), which is a key nutrient sensor that plays an important role in regulating the systemic energy balance [14]. AMPK is activated by alteration of the AMP/ATP ratio, and it induces catabolism to restore the ATP level, which might explain several metabolic effects of metformin, including the shift of major gluconeogenesis substrates from muscle-derived amino acids to other substrates such as lactate produced by anaerobic oxidation of glucose or glycerol released from triglycerides.

The present study had some limitations, since it was a post hoc analysis of a small sample, so definite conclusions cannot be drawn. In addition, muscle strength was not evaluated, so we were unable to assess the influence of the decrease in LM. To overcome these limitations, we are planning a prospective study to confirm the effect of SGLT2 inhibitor + metformin 
therapy (UMIN000030446).

In conclusion, the effect of SGLT2 inhibitors on body composition may differ depending on whether or not patients are also taking metformin. More desirable weight reduction due to preferential fat loss and less muscle loss may be achieved by combining an SGLT2 inhibitor with metformin.

\section{Acknowledgments}

Not applicable.

\section{Financial Disclosure}

Not applicable.

\section{Conflict of Interest}

The authors declare no conflict of interest.

\section{Informed Consent}

Written informed consent was obtained from all patients.

\section{Author Contributions}

YN designed the study, participated in data collection, performed statistical analysis, and wrote the manuscript. HF, SK, $\mathrm{TN}$, and AO participated in data collection and edited the manuscript. YT designed the study and edited the manuscript. All authors have approved the final version to be published.

\section{References}

1. DeFronzo RA, Davidson JA, Del Prato S. The role of the kidneys in glucose homeostasis: a new path towards normalizing glycaemia. Diabetes Obes Metab. 2012;14(1):514.

2. Ohta A, Kato H, Ishii S, Sasaki Y, Nakamura Y, Nakagawa T, Nagai Y, et al. Ipragliflozin, a sodium glucose cotransporter 2 inhibitor, reduces intrahepatic lipid content and abdominal visceral fat volume in patients with type 2 diabetes. Expert Opin Pharmacother. 2017;18(14):14331438.

3. Sha S, Polidori D, Heise T, Natarajan J, Farrell K, Wang $\mathrm{SS}$, Sica D, et al. Effect of the sodium glucose co-transporter 2 inhibitor canagliflozin on plasma volume in patients with type 2 diabetes mellitus. Diabetes Obes Me- tab. 2014;16(11):1087-1095.

4. The National Health and Nutrition Survey in Japan, 2016. in Japanese) http://www.mhlw.go.jp/bunya/kenkou/eiyou/dl/h28-houkoku.pdf. Accessed 14 February 2019.

5. Nagai Y, Ohta A, Sada Y, Kato H, Tanaka Y. Effect of 24week treatment with ipragliflozin on proinsulin/C-peptide ratio in Japanese patients with type 2 diabetes. Expert Opin Pharmacother. 2017;18(1):13-17.

6. Bolinder J, Ljunggren O, Kullberg J, Johansson L, Wilding J, Langkilde AM, Sugg J, et al. Effects of dapagliflozin on body weight, total fat mass, and regional adipose tissue distribution in patients with type 2 diabetes mellitus with inadequate glycemic control on metformin. J Clin Endocrinol Metab. 2012;97(3):1020-1031.

7. Inzucchi SE, Bergenstal RM, Buse JB, Diamant M, Ferrannini E, Nauck M, Peters AL, et al. Management of hyperglycemia in type 2 diabetes, 2015: a patient-centered approach: update to a position statement of the American Diabetes Association and the European Association for the Study of Diabetes. Diabetes Care. 2015;38(1):140149.

8. Inzucchi SE, Bergenstal RM, Buse JB, Diamant M, Ferrannini E, Nauck M, Peters AL, et al. Management of hyperglycaemia in type 2 diabetes, 2015: a patient-centred approach. Update to a position statement of the American Diabetes Association and the European Association for the Study of Diabetes. Diabetologia. 2015;58(3):429-442.

9. International Diabetes Federation Guideline Development Group. Global guideline for type 2 diabetes. Diabetes Res Clin Pract. 2014;104(1):1-52.

10. Haneda M, Noda M, Origasa H, Noto H, Yabe D, Fujita Y, Goto A, et al. Japanese clinical practice guideline for diabetes 2016. J Diabetes Investig. 2018;9(3):657-697.

11. Oishi M, Yamazaki K, Okuguchi F, Sugimoto H, Kanatsuka A, Kashiwagi A, Japan Diabetes Clinical Data Management Study G. Changes in oral antidiabetic prescriptions and improved glycemic control during the years 2002-2011 in Japan (JDDM32). J Diabetes Investig. 2014;5(5):581-587.

12. Ueki K, Sasako T, Kato M, Okazaki Y, Okahata S, Katsuyama H, Haraguchi M, et al. Design of and rationale for the Japan Diabetes Optimal Integrated Treatment study for 3 major risk factors of cardiovascular diseases (J-DOIT3): a multicenter, open-label, randomized, parallel-group trial. BMJ Open Diabetes Res Care. 2016;4(1):e000123.

13. Lee CG, Boyko EJ, Barrett-Connor E, Miljkovic I, Hoffman AR, Everson-Rose SA, Lewis CE, et al. Insulin sensitizers may attenuate lean mass loss in older men with diabetes. Diabetes Care. 2011;34(11):2381-2386.

14. Zhou G, Myers R, Li Y, Chen Y, Shen X, Fenyk-Melody J, Wu M, et al. Role of AMP-activated protein kinase in mechanism of metformin action. J Clin Invest. 2001;108(8):1167-1174. 